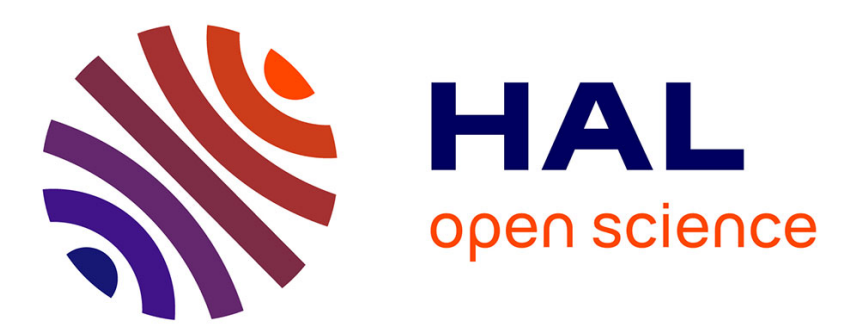

\title{
Conservation et utilisation par les génisses de l'herbe de prairies naturelles. Intérêt des balles rondes enrubannées comparativement à l'ensilage direct et au foin
}

\author{
J-P. Andrieu, C. Demarquilly, J. Rouel
}

\section{- To cite this version:}

J-P. Andrieu, C. Demarquilly, J. Rouel. Conservation et utilisation par les génisses de l'herbe de prairies naturelles. Intérêt des balles rondes enrubannées comparativement à l'ensilage direct et au foin. Productions Animales, 1992, 5 (3), pp.205-212. hal-00895976

\section{HAL Id: hal-00895976 \\ https://hal.science/hal-00895976}

Submitted on 1 Jan 1992

HAL is a multi-disciplinary open access archive for the deposit and dissemination of scientific research documents, whether they are published or not. The documents may come from teaching and research institutions in France or abroad, or from public or private research centers.
L'archive ouverte pluridisciplinaire HAL, est destinée au dépôt et à la diffusion de documents scientifiques de niveau recherche, publiés ou non, émanant des établissements d'enseignement et de recherche français ou étrangers, des laboratoires publics ou privés. 
INRA Prod. Anim., 1992, 5 (3), 205 - 212
J-P. ANDRIEU, C. DEMARQUILLY, J. ROUEL*

INRA Station de recherches sur la

Nutrition des Herbivores

Unité de la Valeur Alimentaire

Theix 63122 Saint-Genès Champanelle

* INRA Domaine d'Orcival

63210 Rochefort-Montagne
Conservation et utilisation par les génisses de l'herbe de prairies naturelles. Intérêt des balles rondes enrubannées comparativement à l'ensilage direct et au foin

(ensilages classiques et foin) : faibles investissements au départ, tant pour la récolte que pour le stockage, chantier de récolte très souple et ne nécessitant que 2 ou 3 personnes, stockage aisé... (ITEB 1990).

Une étude concertée de la faisabilité de l'enrubannage et de la valeur alimentaire et hygiénique des fourrages enrubannés a été entreprise par l'INRA, l'Institut de l'Elevage, l'ITCF, l'ENSAIA et le CEMAGREF dans le cadre d'une convention avec le Ministère de la Recherche et de la Technologie. Nous rapportons ici les résultats de deux années d'étude effectuées sur des génisses laitières à l'INRA d'Orcival. Les résultats des essais en cours sur vaches laitières seront rapportés ultérieurement.

La valeur alimentaire pour des génisses laitières d'ensilages mi-fanés en balles rondes enrubannées a été étudiée par comparaison à celle d'ensilages directs avec ou sans conservateur et du foin correspondants. De plus, avec un ensilage direct sans conservateur, nous avons étudié l'efficacité d'une complémentation en azote peu dégradable. En effet, les essais réalisés sur la conservation et la valeur alimentaire des ensilages directs de prairies naturelles (Andrieu et al 1990) ont montré la perte d'efficacité alimentaire des ensilages directs sans conservateur lorsque les fermentations entraînent une protéolyse trop élevée (N-NH3 > 5\% N total). La valeur PDIE de ces ensilages devient souvent limitante même si la qualité de conservation, satisfaisante par ailleurs, ne diminue pas la quantité ingérée.

\section{Conditions expérimentales}

Les ensilages et foins expérimentaux ont été préparés à partir des mêmes prairies naturelles récoltées aux dates indiquées dans le tableau 1. Nous avons choisi des périodes où les conditions météorologiques étaient favorables à la réalisation d'un foin, aussi les récoltes ont-elles été effectuées par temps beau et chaud. Pour les ensilages directs le 
Tableau 1. Conditions de récolte et doses de conservateurs par tonne de fourrage vert.

\begin{tabular}{|c|c|c|c|}
\hline & Code & $\begin{array}{c}\text { Essai I } \\
1989-90\end{array}$ & $\begin{array}{l}\text { Essai II } \\
1990-91\end{array}$ \\
\hline $\begin{array}{l}\text { Ensilages } \\
\text { Date de récolte } \\
\text { - Sans conservateur } \\
\text { - Acide formique }(620 \mathrm{~g} / \mathrm{l})+\text { formol }(110 \mathrm{~g} / \mathrm{l}) \\
\text { - Caylasil }{ }^{1} \text { (solution) }\end{array}$ & $\begin{array}{l}\mathrm{SC} \\
\mathrm{AFF} \\
\mathrm{CAY}\end{array}$ & $\begin{array}{c}12 \text { juin } 1989 \\
\\
3,21 \\
5,11\end{array}$ & $\begin{array}{c}25 \text { juin } 1990 \\
3,81\end{array}$ \\
\hline $\begin{array}{l}\text { Balles rondes enrubannées } \\
\text { Date de fauche } \\
\text { Date de récolte } \\
\text { Nombre de fanages }\end{array}$ & BRE & $\begin{array}{c}12 \text { juin } 1989 \\
13 \text { Juin } 1989 \\
1\end{array}$ & $\begin{array}{c}25 \text { juin } 1990 \\
26 \text { juin } 1990 \\
1\end{array}$ \\
\hline $\begin{array}{l}\text { Foin } \\
\text { Date de fauche } \\
\text { Date de récolte }\end{array}$ & $\mathrm{F}$ & $\begin{array}{l}12 \text { juin } 1989 \\
15 \text { Juin } 1989\end{array}$ & $\begin{array}{l}23 \text { juin } 1990 \\
26 \text { juin } 1990\end{array}$ \\
\hline
\end{tabular}

${ }^{1}$ Solution Caylasil : 2,49 l d'eau $+2,49 \mathrm{l}$ de mélasse $+55 \mathrm{ml}$ de solution bactérienne et $55 \mathrm{ml}$ de solution enzymatique (Caylasil). L'apport bactérien correspond à un apport de $0,510^{5}$ Lactobacillus Plantarum et $10^{5}$ Streptococcus par gramme de fourrage vert.

fourrage a été finement haché (brins de 15 à $20 \mathrm{~mm}$ ) avec une ensileuse automotrice et stocké dans des silos couloirs ; les conservateurs, acide formique-formol (AFF) et Caylasil + mélasse (CAY), ont été incorporés au niveau de l'ensileuse avec une pompe distributrice. Les balles rondes (BRE) ont été réalisées avec une presse à rouleaux : $1,20 \mathrm{~m} \times 1,20 \mathrm{~m}$ (Welger-Blanchot à chambre fixe) et enrubannées avec un film étirable 'Silotite' large de $50 \mathrm{~cm}$. Le poids moyen des balles a été de $360 \mathrm{~kg}$ (essai I) et $385 \mathrm{~kg}$ (essai II). Quelques lots de deux balles rondes ont été réalisés avant et après la récolte des balles destinées aux génisses pour étudier l'influence de la teneur en matière sèche sur la qualité de conservation. Le foin $(\mathrm{F})$ a été récolté avec une presse à moyenne densité. Le fourrage réservé à chaque traitement a été réparti en plusieurs bandes pour pallier l'hétérogénéité de la parcelle.

Les traitements étudiés suivant les essais sont précisés dans le tableau 1.

Les fourrages ont été distribués durant l'hiver suivant leur préparation à 5 lots de 10 génisses de race laitière (Holstein) comparables par l' âge et le poids dans chaque essai (422 jours et $349 \mathrm{~kg}$ pour l'essai I - 410 jours et $395 \mathrm{~kg}$ pour l'essai II). La période expérimentale a duré respectivement 8 et 11 semaines, mais pendant les périodes préexpérimentales et post-expérimentales (de 2 semaines chacune), les animaux ont reçu un même régime commun (ensilage direct); les gains de poids vif entre ces deux périodes (période d'essai) ont permis de valider les gains réalisés durant la période expérimentale.

Les fourrages ont été distribués à volonté avec pour seule complémentation $100 \mathrm{~g} /$ jour/génisse d'un composé minéral enri- chi en oligo-éléments et en vitamines, excepté dans l'essai II où l' un des deux ensilages directs sans conservateur (SCfp) a été complémenté avec $0,23 \mathrm{~kg}$ MS /jour/génisse de farine de poisson $(68,6 \%$ de matières azotées). Pour chaque lot de génisses en stabulation libre, les quantités distribuées et refusées ont été mesurées 4 jours successifs par semaine. Les animaux ont été pesés deux jours de suite en début et fin des périodes d'essai et d'expérience, et, dans l'intervalle, toutes les 3 semaines.

Par ailleurs, les fourrages ont été distribués à volonté (5 à $10 \%$ de refus) comme seul aliment à six moutons maintenus en cages à métabolisme, afin de déterminer pour chacun d'eux l'ingestibilité et la digestibilité suivant la méthode décrite par Demarquilly et Weiss (1970).

Les critères retenus pour juger de la qualité de conservation des ensilages sont le $\mathrm{pH}$, les proportions d'azote sous forme ammoniacale et soluble et les teneurs en acides gras volatils et alcools. La contamination en spores butyriques et en listéria de ces fourrages (valeur hygiénique) ne sera pas traitée dans cet article.

\section{Résultats}

\section{1 / Composition chimique à la récolte}

Les teneurs en cendres, matières azotées et cellulose brute du fourrage vert sont homogènes entre les traitements dans chaque essai (tableau 2). Elles sont différentes d'un essai à l'autre par suite des stades de végétation différents : stade légèrement plus précoce que le début épiaison pour l'essai I (fauche le 
Tableau 2. Caractéristiques des fourrages.

\begin{tabular}{|c|c|c|c|c|c|c|c|c|c|c|}
\hline & \multicolumn{5}{|c|}{ Essai I } & \multicolumn{5}{|c|}{ Essai II } \\
\hline & $\mathrm{SC}$ & AFF & CAY & BRE & $\mathrm{F}$ & $\mathrm{SC}$ & SCfp & AFF & BRE & $\mathrm{F}$ \\
\hline $\begin{array}{l}\text { Caractéristiques à la récolte } \\
\text { Teneur en MS (\%) } \\
\text { MAT en \% MS } \\
\text { MM en \% MS } \\
\text { CB en \% MS } \\
\text { Gl. solubles en \% MS }\end{array}$ & $\begin{array}{r}19,3 \\
16,4 \\
9,4 \\
24,9 \\
7,3\end{array}$ & $\begin{array}{r}19,0 \\
16,6 \\
8,8 \\
24,0 \\
8,2\end{array}$ & $\begin{array}{r}19,9 \\
16,6 \\
9,0 \\
24,2 \\
8,3 \\
9,1^{2}\end{array}$ & $\begin{array}{r}67,0 \\
15,0 \\
7,8 \\
\text { nd }^{1} \\
\text { nd }\end{array}$ & $\begin{array}{r}82,6 \\
15,4 \\
7,7 \\
\text { nd } \\
\text { nd }\end{array}$ & $\begin{array}{r}18,7 \\
13,8 \\
9,6 \\
27,9 \\
5,7\end{array}$ & $\begin{array}{r}19,1 \\
13,7 \\
9,3 \\
27,9 \\
5,7\end{array}$ & $\begin{array}{r}18,9 \\
13,8 \\
8,9 \\
26,8 \\
6,6\end{array}$ & $\begin{array}{r}50,2 \\
13,8 \\
9,2 \\
28,7 \\
5,9\end{array}$ & $\begin{array}{r}83,1 \\
13,1 \\
8,5 \\
30,1 \\
6,4\end{array}$ \\
\hline $\begin{array}{l}\text { Composition des fourrages } \\
\text { après conservation } \\
\text { Teneur en MS } \text { MS }^{3}(\%) \\
\text { MAT en \% MS } \\
\text { MM en \% MS } \\
\text { CB en \% MS } \\
\text { Gl. solubles résiduels ( \% MS) }\end{array}$ & $\begin{array}{r}22,6 \\
15,6 \\
8,8 \\
25,6 \\
1,0\end{array}$ & $\begin{array}{r}23,2 \\
16,6 \\
8,0 \\
26,1 \\
1,8\end{array}$ & $\begin{array}{r}24,1 \\
15,6 \\
7,9 \\
24,2 \\
1,5\end{array}$ & $\begin{array}{r}66,5 \\
15,7 \\
8,3 \\
25,9 \\
5,8\end{array}$ & $\begin{array}{r}85,5 \\
15,4 \\
8,2 \\
26,0 \\
8,1\end{array}$ & $\begin{array}{r}22,7 \\
13,2 \\
8,1 \\
32,8 \\
0,8\end{array}$ & $\begin{array}{r}22,7 \\
12,9 \\
8,1 \\
32,5 \\
0,8\end{array}$ & $\begin{array}{r}23,0 \\
13,8 \\
8,3 \\
30,5 \\
1,0\end{array}$ & $\begin{array}{r}43,8 \\
14,0 \\
10,1 \\
28,9 \\
0,9\end{array}$ & $\begin{array}{r}84,0 \\
13,9 \\
9,1 \\
29,7 \\
3,2\end{array}$ \\
\hline 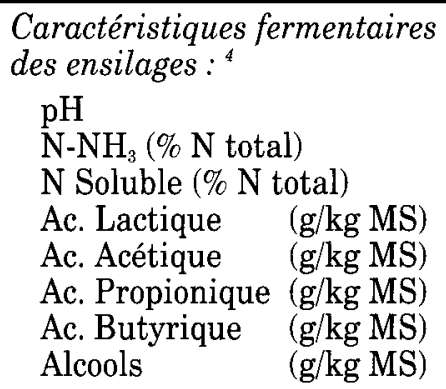 & $\begin{array}{r}4,10 \\
8,3 \\
54,7 \\
63,2 \\
51,1 \\
8,2 \\
2,7 \\
17,8\end{array}$ & $\begin{array}{r}3,72 \\
4,6 \\
47,7 \\
79,0 \\
11,2 \\
0,3 \\
0,1 \\
8,8\end{array}$ & $\begin{array}{r}3,79 \\
6,5 \\
52,6 \\
91,2 \\
40,2 \\
3,5 \\
3,0 \\
13,6\end{array}$ & $\begin{array}{r}5,67 \\
3,0 \\
30,8 \\
10,2 \\
0,8 \\
0,8 \\
0,5 \\
0,6\end{array}$ & & $\begin{array}{r}4,10 \\
9,7 \\
54,5 \\
65,1 \\
39,8 \\
7,0 \\
0,2 \\
12,4\end{array}$ & $\begin{array}{r}4,11 \\
10,3 \\
55,7 \\
65,2 \\
41,3 \\
6,5 \\
1,1 \\
11,5\end{array}$ & $\begin{array}{r}3,84 \\
7,0 \\
49,1 \\
58,0 \\
26,2 \\
0,7 \\
0 \\
8,1\end{array}$ & $\begin{array}{r}5,40 \\
7,8 \\
36,5 \\
0,1 \\
4,5 \\
0,8 \\
4,5 \\
1,0\end{array}$ & \\
\hline
\end{tabular}

${ }^{1}$ nd = non déterminé

mélasse apportée avec le conservateur incluse

${ }^{3}$ Pour les ensilages la matière sèche (MS) a été déterminée à l'étuve $80^{\circ} \mathrm{C}$ et corrigée des pertes de composés volatils selon la méthode de Dulphy et al (1975) ; MAT = Matières azotées totales ; $\mathrm{MM}=$ Matières minérales ; $\mathrm{CB}=\mathrm{Cellulose} \mathrm{brute} \mathrm{;}$ $\mathrm{N}-\mathrm{NH}_{3}=$ Azote ammoniacal $;$ Gl. = Glucides

4 moyenne de 4 échantillons pour chaque ensilage

12 juin) et proche de l'épiaison pour l'essai II (fauche le 25 juin). La teneur en matière sèche à la récolte est peu différente entre essais pour les ensilages directs et le foin, mais elle est supérieure pour les balles rondes de l'essai I et les teneurs en glucides solubles sont plus faibles dans l'essai II par suite d'un rendement en matière sèche beaucoup plus important $(+20 \%)$ en 1990 qu'en 1989 .

\section{2 / Qualité de conservation et valeur nutritive}

La teneur en glucides solubles résiduels est faible pour les ensilages directs. La réduction des fermentations, cas des ensilages mi-fanés, permet de conserver une part non négligeable des glucides solubles dans le fourrage, et ceci d'autant plus que ce dernier est riche en glucides solubles au départ et que la dessication lors du fanage est rapide.

La qualité de conservation des ensilages directs sans conservateur est assez bonne et peu différente entre les deux essais, mais la proportion d'azote ammoniacal $\left(\mathrm{N}-\mathrm{NH}_{3}\right)$ et les teneurs en acide acétique et propionique sont trop élevées. Le mélange acide formique-formol améliore nettement la qualité de conser- vation dans les deux essais, bien que la protéolyse et la teneur en acide acétique soient insuffisamment réduites dans l'essai II. Le conservateur biologique Caylasil associé à une petite quantité de mélasse permet d'obtenir une qualité de conservation intermédiaire entre celle de l'ensilage sans conservateur et avec acide formique-formol.

Les ensilages en balles rondes enrubannées sont bien conservés dans l'ensemble, les teneurs en matière sèche élevées à la récolte $67 \%$ et $50 \%$ respectivement pour les essais I et II) ont réduit d'une part les fermentations productrices d'acides gras volatils et d'alcools et d'autre part la dégradation des protéines du fourrage. Néanmoins, pour l'essai II, on observe une production plus élevée d'acide butyrique et une proportion d'azote sous forme ammoniacale plus importante $(7,8 \%)$; cette orientation fermentaire correspond au développement d'une microflore butyrique protéolytique. L'influence de l'intensité du préfanage et $\mathrm{du}$ mi-fanage sur la réduction des fermentations et l'amélioration de la qualité de conservation est d'ailleurs bien montrée par les caractéristiques fermentaires des quelques balles volontairement récoltées à différentes teneurs en matière sèche (figure 1).
La qualité de conservation des fourrages enrubannés augmente avec leur teneur en matière sèche. 
Figure 1. Qualité de conservation des balles rondes enrubannées selon leur teneur en matière sèche.

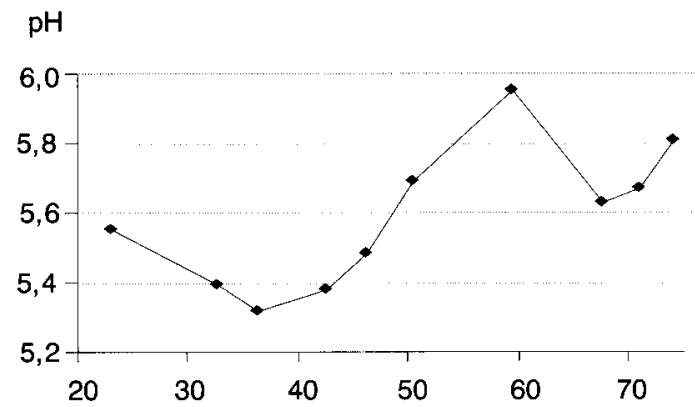

$\% \mathrm{~N}$ total
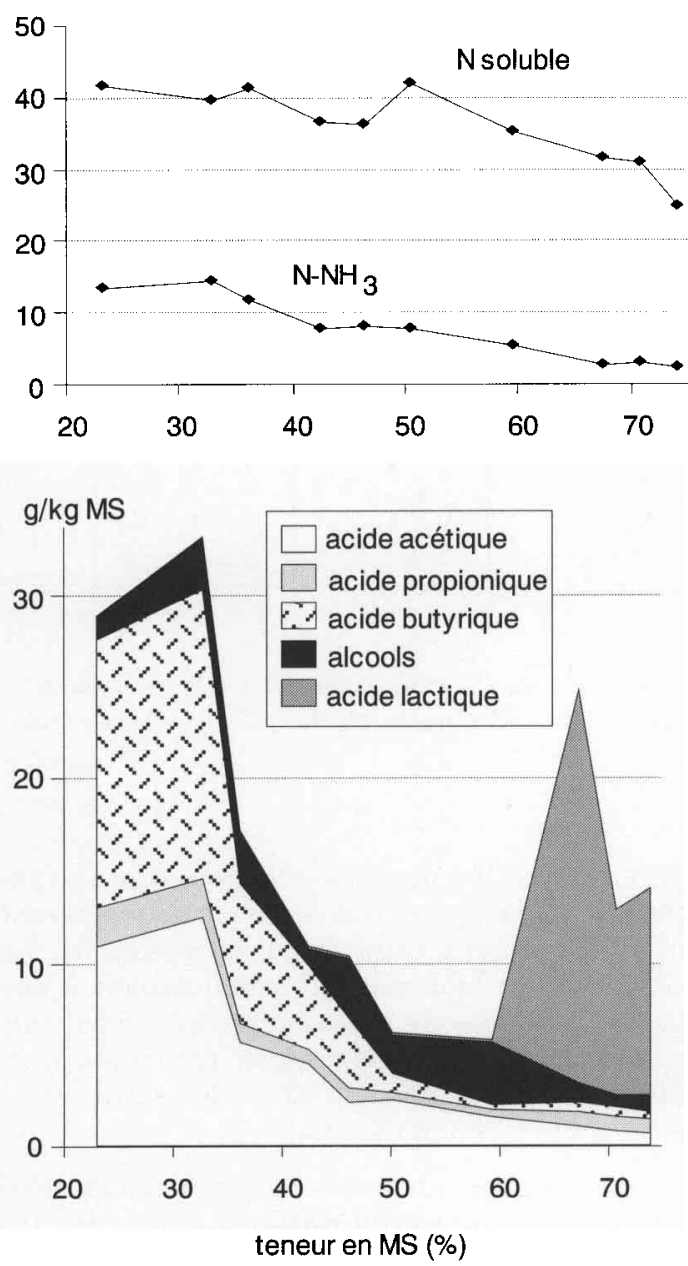

Les fermentations et la protéolyse sont réduites lorsque la teneur en MS à la récolte augmente. La proportion de N-NH3 (exprimées en $\%$ de $\mathrm{N}$ total) diminue lorsque la teneur en matière sèche de l'ensilage augmente $(r=0,96)$.

Les digestibilités de la matière organique (dMO) et des matières azotées (dMAT) sont nettement différentes entre les essais (tableau 3 ) et correspondent bien aux valeurs données dans les tables INRA (1988) pour les fourrages de prairies permanentes de demimontagne récoltés aux stades de végétation indiqués précédemment.

\section{3 / Quantités ingérées et gains de poids des génisses}

Les quantités de fourrage ingérées par $100 \mathrm{~kg}$ de poids vif ont été peu différentes entre traitements à l'intérieur d'un même essai, mais elles sont supérieures dans l'essai I (tableau 4) ce qui est à relier à la date de récolte plus précoce. L'addition de conservateur a eu peu ou pas d'effet sur l'ingestibilité de l'ensilage direct. Dans l'essai II, l'addition de farine de poisson $(0,23 \mathrm{~kg} / \mathrm{jour} /$ génisse $)$ a entraîné une diminution de $0,38 \mathrm{~kg}$ de la quantité de matière sèche d'ensilage ingérée. Comparativement à l'ensilage en balles rondes, le foin a été moins bien ingéré dans l'essai I, mais davantage dans l'essai II. Compte-tenu du dispositif expérimental (contrôle en lots), les différences de quantités ingérées n'ont pu être traitées statistiquement.

Pour les mêmes traitements, les croissances sont supérieures de 200 à $300 \mathrm{~g} / \mathrm{j}$ environ dans l'essai I comparativement à l'essai II (tableau 4). Dans l'essai I, sur la totalité de la période qui prend en compte les périodes pré et post expérimentales pendant lesquelles les animaux ont le même régime, seuls les gains de poids vif du lot ensilage sans conservateur et du lot foin sont significativement plus faibles que ceux réalisés avec l' ensilage Caylasil et les BRE. Dans l'essai II où la durée de la période expérimentale est plus longue, le gain de poids vif réalisé avec l'ensilage sans conservateur durant cette période

Tableau 3. Valeur nutritive des fourrages mesurée sur moutons.

\begin{tabular}{|l|l|l|l|l|l|l|l|l|l|}
\hline & \multicolumn{4}{|c|}{ Essai I } & \multicolumn{4}{c|}{ Essai II } \\
\cline { 2 - 10 } & SC & AFF & CAY & BRE & F & SC & AFF & BRE & F $^{1}$ \\
\hline Digestibilité de la MO (\%) & 74,4 & 71,1 & 72,3 & 73,1 & 67,8 & 64,7 & 63,2 & 63,0 & 64,0 \\
Digestibilité des MAT (\%) & 69,2 & 64,8 & 66,8 & 68,1 & 60,8 & 60,2 & 56,2 & 55,1 & 63,4 \\
UFL/kg MS & 0,94 & 0,88 & 0,90 & 0,91 & 0,84 & 0,76 & 0,73 & 0,72 & 0,73 \\
PDIN/kg MS & 90 & 100 & 94 & 98 & 96 & 74 & 78 & 77 & 89 \\
PDIE/kg MS & 67 & 81 & 75 & 83 & 91 & 57 & 67 & 69 & 85 \\
& & & & & & & & & \\
Quantités ingérées (g MS/kg P0,75) & 54,4 & 60,6 & 56,4 & 59,4 & 81,4 & 46,4 & 47,0 & 47,8 & 47,0 \\
\hline
\end{tabular}

${ }^{1}$ Fauché 2 jours avant les ensilages. 
Tableau 4. Quantités ingérées et gains de poids vif des génisses.

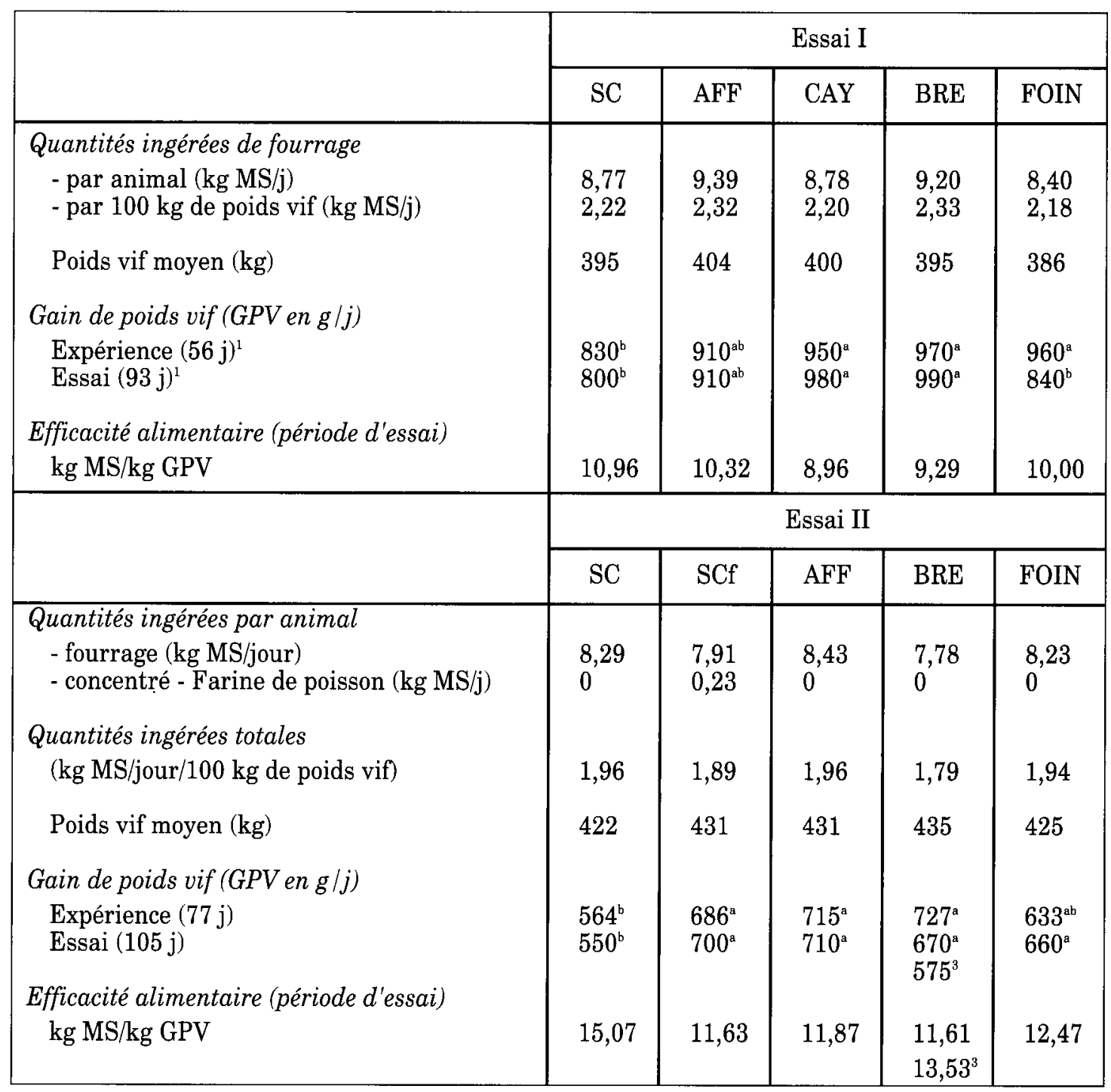

${ }^{1}$ Durée d'expérience et d'essai plus courte pour le lot Foin, respectivement 42 et 79 jours.

${ }^{2}$ Un mois après la fin de l'essai - pesée des animaux en régime herbe.

${ }^{3}$ Valeurs calculées en tenant compte d'une perte de poids supérieure de $10 \mathrm{~kg}$ à la mise à l'herbe par rapport aux autres lots. Cet écart peut signifier une surestimation des gains de poids pendant les périodes d'expérience et d'essai à cause d'un lest digestif plus important.

est significativement plus faible $(P<0,05)$ que ceux réalisés avec les ensilages avec l'acide formique, en balles rondes ou complémenté avec la farine de poisson. Le lot foin, malgré une croissance légèrement plus élevée, n'est pas significativement différent de l'ensilage sans conservateur. Cependant, si pour ce dernier essai on prend en compte les périodes pré et post-expérimentales comme pour l'essai I, qui permettent de mieux tenir compte du lest digestif inter-lots, le gain de poids vif des animaux recevant l'ensilage direct sans conservateur non complémenté est significativement plus faible que celui réalisé par ceux recevant les autres traitements.

Le tableau 5 compare, pour les différents lots de génisses, les apports théoriques nécessaires selon les recommandations INRA (1988) compte tenu de leur poids vif et des gains de poids vif réalisés (période d'essai), aux apports effectifs calculés à partir des quantités de matière sèche ingérées et de la valeur nutritive des ensilages estimée à partir des digestibilités mesurées sur moutons (tableau 3).

On constate que :

- dans l'essai I, les apports effectifs en UFL et PDIE (PDI les plus limitantes) sont nettement supérieurs (respectivement $+24 \%$ et + $33 \%$ en moyenne) aux apports théoriquement nécessaires pour les performances réalisées par les génisses. Ce décalage peut s'expliquer par le fait que la valeur alimentaire élevée des fourrages étudiés et leur haut niveau d'ingestion par les génisses ont probablement conduit à un engraissement des animaux et, par là, à des apports théoriques plus importants que ceux donnés dans les tableaux INRA 1988. Cela a d'ailleurs probablement masqué en partie l'écart de croissance que l'on observe généralement entre un ensilage direct 
Tableau 5. Bilan alimentaire des génisses.

\begin{tabular}{|c|c|c|c|c|c|c|c|c|c|c|}
\hline & \multicolumn{5}{|c|}{ Essai I } & \multicolumn{5}{|c|}{ Essai II } \\
\hline & $\mathrm{SC}$ & AFF & CAY & BRE & FOIN & $\mathrm{SC}$ & SCf & $\mathrm{AFF}$ & BRE & FOIN \\
\hline UFL & & & & & & & & & & \\
\hline Recommandations $^{1}$ & 6,04 & 6,59 & 6,82 & 6,79 & 6,09 & 5,46 & 6,08 & 6,14 & 6,04 & 5,87 \\
\hline Apports & 8,24 & 8,26 & 7,90 & 8,37 & 7,06 & 6,30 & $\begin{array}{r}6,01 \\
+022\end{array}$ & 6,15 & 5,60 & 6,01 \\
\hline $\begin{array}{l}\text { Apports en } \% \text { des } \\
\text { recommandations }\end{array}$ & 136 & 125 & 116 & 125 & 116 & 115 & 102 & 100 & 93 & 102 \\
\hline PDI & & & & & & & & & & \\
\hline Recommandations $^{1}$ & 514 & 537 & 545 & 544 & 514 & 482 & 519 & 522 & 518 & 508 \\
\hline Apports PDIN & 789 & 939 & 825 & 902 & 806 & 613 & $\begin{array}{r}585 \\
+117^{2}\end{array}$ & 658 & 599 & 732 \\
\hline Apports PDIE & 588 & 761 & 659 & 764 & 764 & 473 & $\begin{array}{r}451 \\
+87^{2}\end{array}$ & 565 & 537 & 700 \\
\hline $\begin{array}{l}\text { Apports PDIE en \% des } \\
\text { recommandations }\end{array}$ & 114 & 142 & 121 & 140 & 149 & 98 & 104 & 108 & 104 & 138 \\
\hline
\end{tabular}

Apports recommandés pour les performances de la période d'essai ${ }^{2}$ Apport supplémentaire de la farine de poisson.

avec ou sans conservateur pour des fourrages ayant une valeur alimentaire moins élevée.

- dans l'essai II, le bilan énergétique et azoté des génisses est cohérent entre les apports effectifs et théoriques, et les performances sont différentes entres elles en raison soit de l'apport énergétique, soit de l'apport azoté (PDIE). Ainsi, comme nous l'avions observé dans nos essais en 1987-88 et 1988-89 (Andrieu et al 1990), les croissances réalisées avec l'ensilage direct sans conservateur sont limitées par l'apport de PDIE de l'ensilage. Aussi, l'incorporation d'une petite quantité de farine de poisson qui ne modifie que très peu l'énergie apportée, mais augmente la valeur PDIE de 20\% par kg de MS ingérée (la valeur PDIE est alors proche de celle de l'ensilage avec acide formique) permet d'obtenir une croissance identique à celle réalisée avec l'acide formique. Ce résultat confirme l'importance de la protéolyse dans la détérioration de la valeur azotée des ensilages. Avec les balles rondes enrubannées, les apports énergétiques sont inférieurs aux apports théoriquement nécessaires, mais ils ne le sont plus si l'on abaisse la croissance journalière de $95 \mathrm{~g}$ (valeur estimée à partir de la chute de poids à la mise à l'herbe de $10 \mathrm{~kg}$ plus élevée que celle des autres lots). Pour les ensilages directs avec un complément de farine de poisson ou avec acide formique-formol et pour le foin les performances semblent limitées essentiellement par les apports énergétiques.

\section{Discussion}

Ces deux essais mettent d'abord en évidence l'influence primordiale de la date de récolte du fourrage sur les croissances des génisses (904 g/j en moyenne en 1989 contre $658 \mathrm{~g} / \mathrm{j}$ en 1990), influence nettement plus importante que celle entraînée par les traitements étudiés à l'intérieur d'un essai donné. Ils montrent en outre l'intérêt de l'emploi des conservateurs efficaces pour améliorer la valeur azotée des ensilages directs et permettent de situer la valeur alimentaire des balles rondes enrubannées par comparaison à celle des ensilages classiques et des foins.

\section{1 / Qualité de conservation et valeur azotée des ensilages}

Dans l'essai II, l'apport d'azote non dégradable dans le rumen (farine de poisson) en complémentation de l'ensilage direct sans conservateur permet d'obtenir un gain de poids proche de celui obtenu avec des conservateurs efficaces, et cela bien que la quantité ingérée d'ensilage n'ait pas augmenté contrairement à ce qui a parfois été observé avec des ensilages mal conservés, conduisant à un mauvais "état azoté" des animaux. Ce n'est pas le cas dans notre étude puisque l'ensilage sans conservateur distribué sans complémentation permet déja une croissance journalière de $550 \mathrm{~g} / \mathrm{j}$. Sa complémentation par de la farine de poisson améliore cependant l'efficacité alimentaire de plus de $30 \%$ et permet une croissance comparable à celle obtenue avec l'ensilage préparé avec l'addition d'acide formique-formol. De même, Waldo et Tyrell (1980) avaient obtenu une amélioration du gain de poids vif des bouvillons sans accroître les quantités d'énergie digestible ingérées, en remplaçant un ensilage sans conservateur par un ensilage traité par un mélange d'acide formique-formol à la mise en silo, ou en le complémentant avec de la caséine protégée. Cela démontre bien que les performances des animaux recevant sans complémentation des ensilages d'herbe préparés sans conservateur sont souvent plus limitées par la valeur azotée de ces ensilages que par leur valeur énergétique (Andrieu et al 1990). 
L'emploi de bons conservateurs à la mise en silo s'avère cependant souvent plus efficace que l'addition d'azote peu dégradable lors de la distribution car il augmente non seulement la valeur azotée de l'ensilage mais le plus souvent aussi la quantité ingérée bien que ce n'ait pas été le cas dans cet essai. A cet égard l'utilisation du conservateur Caylasil (mélange de ferments lactiques et d'enzymes cellulolytiques) associé à une petite quantité de mélasse s'avère quasiment aussi efficace que le mélange acide formique-formol pour améliorer la conservation de fourrage pas trop déficitaire en glucides solubles ( $8 \%$ de la MS). Il permet le même gain de poids vif.

L'amélioration de la qualité de conservation peut aussi être obtenue par un préfanage poussé. Les résultats présentés à la figure 1 montrent clairement que la qualité de conservation des balles rondes enrubannées s'améliore lorsque la teneur en matière sèche augmente et qu'il faut dépasser $50 \%$ de MS pour que la proportion d'azote sous forme ammoniacale soit inférieure ou égale à $5 \%$ et corresponde alors à celle d'excellents ensilages. Ce résultat est confirmé par ceux obtenus par Weddel (1990) et Haigh et Peers (1990). A des teneurs en matière sèche supérieures à $50 \%$, les balles rondes enrubannées contiennent très peu de produits de fermentation (acide lactique, acides gras volatils et alcools) d'une part, et d'autre part, peu de spores butyriques comme le montrent les dénombrements effectués par l'Institut de l'Elevage et dont les résultats devraient être publiés prochainement.

\section{2 / Comparaison de la valeur alimentaire : balles rondes enrubannées/ ensilages directs/foin}

Dans l'essai I les BRE ont été ingérées en quantité équivalente à l'ensilage à l'acide formique-formol et en quantité un peu supérieure aux autres traitements. Dans l'essai II en revanche, elles ont été ingérées en quantité inférieure aux autres traitements. Cette ingestion plus faible ne semble pas résulter d'une teneur en matière sèche plus faible $(43,8 \%)$ dans l'essai II par comparaison à l'essai I $(67,0 \%)$ puisque elle n'a pas été observée (Andrieu et al 1981, Dulphy et al 1984) dans les essais précédents avec des balles rondes conservées en sacs ou en meules et ayant une teneur en matière sèche équivalente (respectivement 43,6 et $42,4 \%$ ), ni par E. Daburon (communication personnelle) dans un essai récent comparable aux nôtres avec des BRE à 40,1 \% de MS. Elle résulte peut être du développement de moisissures dans les trois dernières semaines de l'essai, et (ou) d'une contamination par de la terre, variable suivant les balles. La teneur moyenne en cendres des ensilages en BRE $(10,1 \%)$ est en effet supérieure à celle des autres ensilages $(8,1$ à $8,3 \%)$, ce qui expliquerait aussi la quantité non négligeable d'acide butyrique trouvée dans ces BRE malgré une teneur en MS de $43,8 \%$.

Dans les essais I et II, les performances des génisses, notamment si on considère la période d'essai plus fiable car d'une durée plus longue que la période expérimentale proprement dite, sont avec les BRE équivalentes à celles avec l'acide formique - formol et supérieures à celles avec l'ensilage direct et avec le foin (non significativement dans l'essai II, mais le GMQ avec le foin aurait vraisemblablement été de l'ordre de $600 \mathrm{~g} / \mathrm{j}$ si la fauche du foin avait été effectuée le même jour que celle des BRE).

L'efficacité alimentaire exprimée en $\mathrm{kg}$ de matière sèche ingérée pour obtenir $1 \mathrm{~kg}$ de gain de poids est, avec les BRE, équivalente à celle des ensilages avec conservateur et est légèrement supérieure à celle des foins récoltés dans d'excellentes conditions.

En définitive la valeur alimentaire des fourrages en balles rondes enrubannées récoltées dans d'excellentes conditions climatiques (pressage le 2ème jour après la fauche) s'avère supérieure ou égale à celle des foins, eux mêmes récoltés dans d'excellentes conditions (pressage le 4ème jour après la fauche) et équivalente à celle des ensilages récoltés en coupe directe avec addition à la récolte de conservateur efficace. Ces excellentes conditions climatiques ne sont pas toujours obtenues au printemps, surtout dans certaines régions où le temps est particulièrement instable au stade optimum de récolte, comme ce fut le cas pour l'essai II, où la récolte a du être retardée de 10 jours par comparaison à 1989 (année aux conditions climatiques tout à fait exceptionnelles) mais avec des conséquences très nettes sur les performances dè croissance des génisses. Aussi, il ne nous semble pas raisonnable de préconiser aux éleveurs ayant à récolter au printemps une grande quantité de fourrages à leur stade optimum (apparition des premiers épis) pour l'alimentation hivernale d'animaux à besoins élevés (vaches laitières) de remplacer l'ensilage classique en coupe directe ou simplement ressuyé par des fourrages pressés en BRE. En revanche les BRE semblent mieux adaptées aux zones ou l'alimentation hivernale des animaux reste à base de foins (vaches allaitantes par exemple). L'intérêt des BRE est alors de pouvoir prendre le risque de commencer les récoltes à un stade plus précoce, les séquences de beau temps de 2-3 jours nécessaires pour les BRE étant plus fréquentes que celles de 45 jours nécessaires pour le foin, et leur prévision beaucoup plus fiable. Si le beau temps se prolonge, les fourrages à base de graminées pourront être récoltés sous forme de foin car les différences de valeur alimentaire entre les $\mathrm{BRE}$ récoltées entre 55 et $65 \%$ de matière sèche et le foin récolté entre 80 et $85 \%$ sont trop faibles pour compenser le coût de l'enrubannage (environ $20 \mathrm{FF}$ par balle). Il doit cependant en être autrement avec les fourrages à base de légumineuses pour lesquels les différences de valeur alimentaire doivent

\section{L'efficacité alimentaire des fourrages enrubannés récoltés dans d'excellentes conditions est voisine de celle des ensilages avec conservateur et supérieure à celle des ensilages sans conservateur et du foin.}


être beaucoup plus élevées par suite des pertes importantes de feuilles dès que la teneur en matière sèche au ramassage dépasse $60 \%$. Enfin quelle que soit la région, les $\mathrm{BRE}$ sont intéressantes pour récolter, quand cela est nécessaire, des petites quantités de fourrages (excédents de paturage) évitant ainsi la mise en oeuvre d'un chantier de récolte important (machines, silos, personnels) inadapté à ces petites quantités.

\section{Références bibliographiques}

Andrieu J.P., Gaillard F., Dulphy J.P., 1981, L'ensilage d'herbe mi-fané en balles rondes, utilisation par des génisses laitières de 1 an. Bull. Tech. CRZV Theix. INRA, 46, 31-37

Andrieu J.P., Demarquilly C., Rouel J., 1990. Conservation et valeur alimentaire des ensilages directs de prairies naturelles. Comparaison de trois types de conservateur. INRA Prod. Anim., 3 (1), 67-73.

Demarquilly C., Weiss P., 1970. Tableaux de la valeur alimentaire des fourrages verts. Etude S.E.I. No ${ }^{\circ}$ 2. Ed. INRA, Versailles.

Dulphy J.P., Gaillard F., Garel J.P., Andrieu J.P., Rouel J., 1984. Etude de la valeur alimentaire d'ensilages mifanés distribués à des génisses et à des vaches laitières Cas des fourrages récoltés avec une remorque autochargeuse ou une presse à grosses balles. Bull. Tech. CRZV Theix, INRA, 57, 5-12.

Haigh P.M., Peers D.G., 1990. The effect of dry matter content on the fermentation of big bale grass silages made on commercial farms in North and South Wales
1984-88. In the Proceedings of the Ninth Silage Conference - Faculty of Agriculture, Published by the University of Newcastle upon Tyne.

INRA, 1988. Alimentation des bovins, ovins et caprins, Ed. R. Jarrige, INRA Publications, Route de St-Cyr, 78000 Versailles.

ITEB, 1990. Les éleveurs et les balles rondes enrubannées. Enquête de motivation $N^{\circ} 90081$. 149, Rue de Bercy - 75595 Paris Cedex 12.

Waldo D.A., Tyrell H.F., 1980. The relation of insoluble nitrogen intake to gain, energy retention and nitrogen retention in Holstein steers. In Proc. 3rd European Association for animal production on protein metabolism and nutrition. Ed. H.I. Oslage and K. Rohr. EAAP Publication $\mathrm{N}^{\circ}$ 27, 572-577.

Weddel J.R., 1990. The effects of a bacterial inoculant additive on big bale silage. In the Proceedings of the Ninth Silage Conference - Faculty of Agriculture, Published by the University of Newcastle upon Tyne.

\section{Summary}

Conservation and utilization of grass from natural pastures. The advantages of wrapped round bales as compared to silage and hay

The conservation of wilted and heavy wilted grass pressed into round bales which are then wrapped has developed rapidly in France and Northern Europe since the recent development of quality stretchable plastic films and of machines which automatically wrap the bales.'

The feeding value of this type of forage for one year old dairy heifers was compared during two successive winters to that of the corresponding direct precision-chopped silage, with or without the addition of additives, and that of the corresponding hay.

The fermentation quality of this wrapped silage increases with dry matter content and becomes excellant when this content is greater than $50-55 \%$.
Under these conditions the feeding value intake, consumption index is the equivalent of that of silage with formic acid and is slightly greater than that of hay harvested in good conditions and much greater thant that of silage without additive.

These experiments have demonstrated the interest of grass from natural pasture, of an early harvest (early earing) of a additive based on lactic bacteria and cellulolytic enzymes, associated with a small quantity of molasses, and of the distribution of a small amount of nitrogen which is almost undegradable to animals given silage prepared without additive.

ANDRIEU J.-P., DEMARQUILLY C., ROUEL J. 1992. Conservation et utilisation par les génisses de l'herbe de prairies naturelles. Intérêt des balles rondes enrubannées comparativement à l'ensilage direct et au foin. INRA Prod. Anim., 5 (3), $205 \cdot 212$. 